\title{
New approach to interstage care for palliated high-risk patients with congenital heart disease
}

\author{
Nancy C. Dobrolet, MD, Jo Ann Nieves, MSN, CPN, ARNP, PNP-BC, Elizabeth M. Welch, MD, \\ Danyal Khan, MD, Anthony F. Rossi, MD, Redmond P. Burke, MD, and Evan M. Zahn, MD
}

\begin{abstract}
Objective: Home surveillance monitoring might identify patients at risk for interstage death after stage 1 palliation for hypoplastic left heart syndrome. We sought to identify the effect that a high-risk program might have on interstage mortality and identification of residual/recurrent lesions after neonatal palliative operations.
\end{abstract}

\begin{abstract}
Methods: Between January 2006 to January 2010, newborns after stage 1 palliation for hypoplastic left heart syndrome or shunt placement were invited to participate in our high-risk program. Patients enrolled in our high-risk program comprise the study group. Patients who had similar operations between January 2002 and December 2005 comprise the control group. Comparisons are made between the 2 groups with respect to interstage mortality and the frequency and timing of interstage admissions requiring medical, catheter, or surgical treatment.
\end{abstract}

Results: Seventy-two patients met the criteria for our high-risk program. Fifty-nine (82\%) of 72 patients were enrolled. Among 19 patients with hypoplastic left heart syndrome in our high-risk program, outpatient interstage mortality was zero. Outpatient interstage mortality for the 36 control subjects with hypoplastic left heart syndrome was $6 \%$. Among 40 patients with shunts in the study group, there was 1 outpatient interstage death compared with $4(6 \%)$ deaths in 68 subjects in the control group. Significant residual/recurrent lesions were identified with similar frequency between the 2 groups. However, after shunt operations, these lesions were detected and treated at significantly younger mean ages for patients followed in the high-risk program $(P<.005)$.

Conclusions: Initiation of a high-risk program might decrease interstage mortality after high-risk neonatal palliative operations. Such an approach might contribute to earlier detection of significant residual/recurrent lesions amenable to therapy. (J Thorac Cardiovasc Surg 2011;142:855-60)

Survival to discharge has significantly improved after stage 1 palliation (S1P) for infants born with hypoplastic left heart syndrome (HLHS); however, interstage mortality remains an important problem, with $10 \%$ to $15 \%$ interstage attrition reported. ${ }^{1-5}$ Recently, implementation of home surveillance monitoring (HSM), which is the use of home pulse oximetry and daily weights, has been reported to result in improved identification of patients at risk, thereby allowing for early surgical or catheter intervention. ${ }^{6}$ We hypothesized that other neonates who undergo palliative surgical procedures might benefit from such monitoring. Additionally, we believed that the formation of a high-risk program designed specifically to closely monitor, track, and treat these patients might offer improved detection of important residual/recurrent lesions and thereby contribute to improved interstage survival.

The purpose of this report is to describe our experience with this approach since its inception at our center. Our cen-

\footnotetext{
From the Congenital Heart Institute at Miami Children's Hospital and Arnold Palmer Children's Hospital, Miami, Fla.

Disclosures: Authors have nothing to disclose with regard to commercial support.

Received for publication Sept 15, 2010; revisions received Dec 16, 2010; accepted for publication Jan 25, 2011; available ahead of print March 14, 2011.

Address for reprints: Nancy C. Dobrolet, MD, Miami Children's Hospital, Cardiology, 3200 SW 60th Court, Miami, FL 33155 (E-mail: nancy.dobrolet@mch.com). $0022-5223 / \$ 36.00$

Copyright (C) 2011 by The American Association for Thoracic Surgery doi:10.1016/j.jtcvs.2011.01.054
}

ter is unique in that it is located in a tertiary care children's hospital with no birthing center or affiliation with any neonatal units. Therefore there are no live births at our institution, and our patients are $100 \%$ referred from outside cardiology groups with no formal affiliation with our hospital.

Before the initiation of our high-risk program, all interstage care was provided by the individual referring cardiology groups. Typically, the patient would not return for evaluation by our cardiac team until the time of the prestage 2 (S2) catheterization or in the event of an interstage admission.

\section{MATERIALS AND METHODS \\ Patient Selection/Inclusion}

Beginning in January 2006, a high-risk program was formed (see below) at our tertiary care institution for the express purpose of providing systematic, closely monitored care of infants believed to be at high risk after palliative neonatal procedures. Our high-risk program was designed to include a high-risk outpatient clinic coupled with HSM. Enrollment in the program was offered to those families whose infants underwent S1P for HLHS or HLHS variants or placement of a Blalock-Taussig (BT) or central shunt with or without a Damus-Kaye-Stansel (DKS) shunt.

To identify a control group, we performed a retrospective review of all infants with similar anatomic diagnoses who underwent similar operations during the previous 4 years (January 2002-December 2005). Interstage care varied for these patients, depending on the practice of the different referring cardiology groups. 


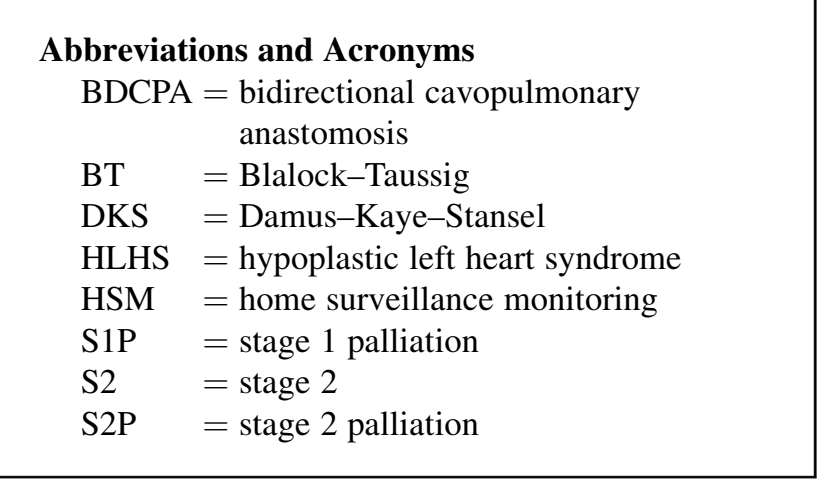

Patients' records were carefully reviewed for the occurrence of unplanned interstage hospital admissions requiring (1) inotropic support, (2) catheter-based interventions to alleviate recurrent/residual lesions, (3) surgi$\mathrm{cal}$ intervention to alleviate recurrent/residual lesions and early progression to stage 2 palliation (S2P), or (4) need for cardiopulmonary bypass support.

Patients after S1P for HLHS or shunt placement with or without a DKS shunt were deemed at high risk for interstage morbidity and mortality. In January 2006, we began to follow these patients in our high-risk program, which included a high-risk outpatient clinic visit and HSM. The details of our high-risk program are described below. We were able to compare interstage mortality and interstage admissions requiring interventions for patients before and after the inception of our high-risk program.

This study was approved by the institutional review board. The same surgeons performed all operations during the 2 time periods using similar techniques, and patients in both groups were discharged on $20 \mathrm{mg} / \mathrm{d}$ oral aspirin.

\section{Home Surveillance Monitoring}

Similar to other approaches for discharging high-risk neonates, ${ }^{7}$ each patient was discharged after detailed parental education related to symptoms and signs to monitor and written guidelines instructing when to contact their private cardiologist and the high-risk team. Families were instructed to measure and record daily oxygen saturations and daily weights using their home pulse oximeter and digital scale. Parents were also instructed on the preparation of a 24 calories per ounce formula. Reportable signs for parents thought to relate to compromise in cardiopulmonary or nutritional status are shown in Figure 1. Parents were supplied with a medical passport, which listed their infant's anatomy and surgical procedures and expected oxygen saturation level. Printed materials supplied to each family at the time of discharge are listed in Figure 2. Each referral pediatrician and cardiologist was contacted by means of fax and telephone to review the plan of care, reportable symptoms, and resources available for consultation 24 hours per day.

The high-risk cardiac nurse practitioner contacted families by telephone every other week or more frequently if the infant had issues to review home monitoring data, weight gain, formula preparation, access to their primary cardiologist and pediatrician, general development, medication administration, and follow-up with specialty physicians, if indicated. In addition, attention was given to the identification of any pending insurance coverage issues. Our electronic medical record, I-Rounds (Teges, Coral Gables, Fla), was used to track all telephone conversations. Patients participating in the high-risk program were flagged for easy identification and followup. Electronic high-risk progress notes were entered by the cardiac nurse practitioner after all telephone communications with families, primary pediatricians/cardiologists, or both. These notes typically contained the home oxygen saturation levels and weight gain data and could be reviewed at any time by any member of our cardiac team.

\section{FAMILY INFORMATION}

Your baby has Hypoplastic Left Heart Syndrome. A Norwood operation was completed with placement of a shunt to provide blood flow to the lungs

${ }^{* *}$ Expected oxygen saturation levels will be about $75-85 \%$.

CALL your Cardiologist or the Miami Children's Hospital Cardiac Care Center if your baby has

-Poor feeding

-Weight loss or no weight gain in 3 days

-Breaths fast or hard

- Oxygen levels are are $75 \%$ or less, or above $90 \%$

-Appears blue or pale in the face, lips or hands while at rest

- Diarrhea or vomiting, sweating

-Irritable, fussy

-If it necessary to hospitalize your baby

Primary Cardiologist: Dr.

Phone:

24 HOURS/Day CONTACT: Miami Children's Hospital Cardiac ICU 3056696500 or 18006664278

Miami Children's Hospital Cardiology 7866244344 (Jo Ann Nieves ARNP) or pager 3052867222

If you feel you have an Emergency Call 911

FIGURE 1. Example of printed material for the family of a patient with HLHS.

\section{High-Risk Outpatient Clinic}

All outpatient encounters took place at the Congenital Heart Institute at Miami Children's Hospital. Before discharge, families were scheduled to return to our institution for their first high-risk appointment 1 month after discharge (Figure 3). Our clinic did not replace regularly scheduled visits with the primary cardiologist. Our high-risk team included an intensive care cardiologist and cardiac nurse practitioner, who evaluated all patients enrolled in the program. This outpatient encounter included a sedated echocardiogram, chest radiograph, electrocardiogram, and physical examination by the high-risk team. If at the time of discharge the pulmonary artery anatomy was of potential concern, quantitative nuclear medicine lung perfusion scans were performed as well. The clinic evaluation included meticulous review of all medication and formula preparation and administration. Home monitoring data were reviewed with the parents. All echocardiograms performed in the high-risk clinic were interpreted by our Congenital Heart Institute echocardiographers.

- Laminated wallet card with following information: - Name/number of primary cardiologist, pediatrician and $\mathrm{CICU}$ - Name of congenital heart defect and operation performed - Expected oxygen saturation level

-Expected daily weight increase for each infant

-24 calorie/oz formula preparation instructions with expected intake -Infection control guidelines-hand washing, screening contacts -Synagis explanation with clinic site closest to family -Developmental screening and support contact list for Florida -Prescription written for Early Steps evaluation at 4 months of age - Normal infant developmental milestones

-Websites for information about congenital heart disease

-Preprinted forms to record weights/saturations/heart rates/oral intake

- Home care booklet with information about wound care etc.

-Descriptions of the upcoming surgical procedures for each patient

FIGURE 2. Materials provided to families at discharge 


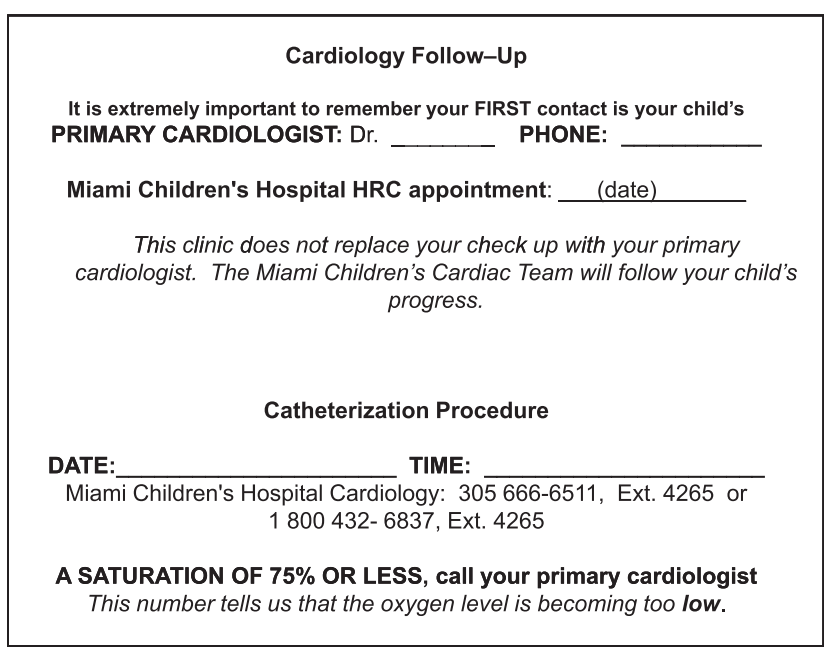

FIGURE 3. Example of a high-risk clinic appointment card.

It was determined by the high-risk team whether the patient's clinical course was progressing as expected. If there were no problems detected, patients were scheduled for their pre-S2 catheterization. For our patients with HLHS, this was between 3 and 4 months of age. The second palliative operation was scheduled soon after completion of the pre-S2 catheterization, typically by $4 \frac{1}{2}$ months of age for patients with HLHS. HSM continued until the time of the second palliative operation.

If during the high-risk visit a recurrent/residual lesion was suspected, the patient was scheduled for further evaluation, usually cardiac catheterization. If a significant decrease in cardiac function was detected, the patient was directly admitted to the cardiac intensive care unit for further evaluation, treatment, and intervention when indicated. In selected cases additional high-risk clinic appointments were scheduled for follow-up of a mild residual lesion or as follow-up to an interstage intervention.

\section{Statistical Analysis}

Our prospective sequel server database, I-Rounds, was used for data collection. The database allows for identification of all palliated patients with congenital heart disease and provides a review of all hospital admissions, interstage interventions/operations, and mortality data.

The Statistical Package for Social Sciences (PASW 18; SPSS, Inc, Chicago, Ill) was used to organize, validate, and analyze the collected data. Indicators of central tendency and dispersion (medians, means, standard deviations, standard errors of the mean, and $95 \%$ confident intervals) were estimated for quantitative variables, whereas frequencies and percentages were used for qualitative variables. The Mann-Whitney $U$ test was selected to compare median values between groups. We also used the $\chi^{2}$ test or Fisher's exact test when appropriate to identify differences between proportions or associations between categorical variables. Tables were constructed to present the most relevant findings.

\section{RESULTS}

The study group consisted of 59 patients followed in our high-risk program. Control subjects consisted of 104 patients before the inception of our high-risk program. The patients' diagnoses are listed in Table 1. The operative characteristics are listed in Table 2.

\section{Interstage Mortality}

For more information on interstage mortality, see Table 3.
TABLE 1. Patients' diagnoses

\begin{tabular}{lcc}
\hline & $\begin{array}{c}\text { Study group } \\
(\mathbf{n}=\mathbf{5 9})\end{array}$ & $\begin{array}{c}\text { Control group } \\
(\mathbf{n}=\mathbf{1 0 4})\end{array}$ \\
\hline S1P-HLHS & 19 & 36 \\
AA with MS or MA & 13 & 20 \\
AS with MS or MA & 4 & 7 \\
HLHS with VSD & 1 & 7 \\
HLHS with intact atrial septum & 1 & 0 \\
HLHS with TAPVC & 0 & 2 \\
Shunt & 40 & 68 \\
With DKS shunt & & \\
DORV with subAS & 2 & 3 \\
Unbalanced AVC & 0 & 2 \\
Interrupted aortic arch, VSD & 1 & 3 \\
Shone-type complex & 3 & 1 \\
TGA variant & 3 & 3 \\
DILV & 1 & 2 \\
Without DKS shunt & & \\
Pulmonary atresia, VSD & 8 & 12 \\
Pulmonary atresia, IVS & 8 & 11 \\
Heterotaxy & 6 & 12 \\
Ventricular inversion & 0 & 3 \\
Tricuspid atresia & 6 & 7 \\
Unbalanced AVC & 0 & 1 \\
Truncus arteriosus, IAA & 0 & 1 \\
TOF/DORV variants & 3 & 0 \\
Interrupted aortic arch, VSD & 0 & 1 \\
DILV & 1 & \\
\hline SIP, Sta I pall & & \\
\hline
\end{tabular}

SIP, Stage 1 palliation; $H L H S$, hypoplastic left heart syndrome; $A A$, aortic atresia $M S$, mitral stenosis; $M A$, mitral atresia; $A S$, aortic stenosis; $V S D$, ventricular septal defect; TAPVC, total anomalous pulmonary venous connection; DKS, DamusKaye-Stansel; DORV, double-outlet right ventricle; $A V C$, atrioventricular canal; $T G A$, transposition of the great arteries; $D I L V$, double-inlet left ventricle; $I V S$, intact ventricular septum; IAA, interrupted aortic arch; TOF, tetralogy of Fallot; Shone-type complex, multiple left-sided hypoplastic lesions not amenable to 2-ventricle repair.

Study group. SIP for HLHS. No patients in our high-risk program have died at home after S1P for HLHS. One patient died in the hospital after readmission for methicillin-resistant

TABLE 2. Operative variables

\begin{tabular}{lccl}
\hline & $\begin{array}{c}\text { Study } \\
\text { group }\end{array}$ & $\begin{array}{c}\text { Control } \\
\text { group }\end{array}$ & $\begin{array}{c}\boldsymbol{P} \\
\text { value }\end{array}$ \\
\hline S1P-HLHS and variants & & & \\
$\quad$ Sex (male) & $73 \%$ & $57 \%$ & NS \\
$\quad$ Birth weight (kg) & $3.2 \pm 0.1$ & $3.2 \pm 0.1$ & NS \\
Age at operation (d) & $5 \pm 1$ & $12 \pm 2$ & $<.05$ \\
Length of stay (d) & $34 \pm 5$ & $30 \pm 3$ & NS \\
Perfusion time (min) & $219 \pm 7$ & $243 \pm 8$ & NS \\
DHCA (min) & $18.6 \pm 1.8$ & $17.9 \pm 2.6$ & NS \\
Shunts & & & \\
Sex (male) & $55 \%$ & $52 \%$ & NS \\
Birth weight (kg) & $3.2 \pm 0.2$ & $3.3 \pm 0.1$ & NS \\
Median age at operation (d) & $4(0-37)$ & $6(1-67)$ & NS \\
Length of stay (d) & $33 \pm 4$ & $33 \pm 5$ & NS \\
\hline
\end{tabular}

Variables are reported as means \pm standard errors of the mean unless otherwise noted $S I P$, Stage 1 palliation; $H L H S$, hypoplastic left heart syndrome; $N S$, not statistically significant; $D H C A$, deep hypothermic circulatory arrest. 
TABLE 3. Interstage mortality for patients undergoing S1P procedures with shunts

\begin{tabular}{|c|c|c|c|c|c|c|}
\hline & Patients (n) & $\begin{array}{c}\text { Survival to } \\
\text { discharge }\end{array}$ & $\begin{array}{c}\text { Enrolled } \\
\text { high-risk } \\
\text { program }\end{array}$ & $\begin{array}{c}\text { Interstage } \\
\text { home death* }\end{array}$ & $\begin{array}{c}\text { Interstage } \\
\text { inpatient death* }\end{array}$ & $\begin{array}{c}\text { S2P } \\
\text { pending }\end{array}$ \\
\hline \multicolumn{7}{|c|}{ Study group } \\
\hline S1P & 26 & $25(96 \%)$ & $19(76 \%)$ & 0 & $1(5 \%)$ & 0 \\
\hline Shunts & 53 & $47(89 \%)$ & $40(85 \%)$ & $1(3 \%)$ & 0 & 0 \\
\hline \multicolumn{7}{|c|}{ Control group } \\
\hline S1P & 43 & $36(84 \%)$ & - & $2(6 \%)$ & $2(6 \%)$ & 0 \\
\hline Shunts & 74 & $68(92 \%)$ & - & $4(6 \%)$ & 0 & 0 \\
\hline
\end{tabular}

$S 2 P$, Stage 2 palliation; $S 1 P$, stage 1 palliation. *Not significant.

Staphylococcus aureus-induced endocarditis with resultant shunt thrombosis.

Shunts. There was 1 outpatient death in an international patient after placement of a BT shunt for an unbalanced atrioventricular canal defect. This patient died 5 days after discharge. Because of the lack of adequate insurance coverage, this patient was unable to be discharged with a pulse oximeter, and the patient had no immediate access to a pediatric cardiologist in the location where the family resided. No autopsy was performed.

Control group. SIP for HLHS. Four patients died after S1P for HLHS in this group. Two patients died suddenly at home. One died 28 days after discharge, having been admitted 3 weeks before with an episode of dehydration. The other patient died at 5 months of age with a history of poor compliance with follow-up. Neither patient had associated chromosomal or other anomalies. Autopsies were not performed.

There were 2 inpatient deaths in patients with HLHS and pulmonary vein stenosis. One patient died after readmission and a surgical attempt at relief of the pulmonary venous obstruction, and the other patient died shortly after readmission for severe cyanosis and pulmonary venous obstruction.

Shunts. Four patients died suddenly at home after shunt operations. One patient with heterotaxy syndrome died 2 days after discharge. This patient's hospital course was significant for shunt thrombosis, requiring stenting of the shunt before discharge. One international patient with pulmonary atresia after placement of a BT shunt, died of unknown cause 5 months after discharge. One patient with pulmonary atresia and ventricular septal defect died at 5 months of age after being home for 2 months. The final patient died after shunt and DKS anastomosis for an interrupted aortic arch and ventricular septal defect. This patient also had $22 \mathrm{q}$ deletion.

\section{Identification of Interstage Recurrent/Residual Lesions Requiring Treatment}

S1P. In the study group residual lesions identified after S1P for HLHS included recurrent arch obstruction $(n=6)$, restrictive atrial communication $(\mathrm{n}=1)$, and severe right ventricular dysfunction $(n=5)$. In the control group lesions identified included recurrent arch obstruction $(\mathrm{n}=7)$ and left pulmonary artery stenosis $(n=1)$. The mean ages at detection, admission, and treatment of these lesions were not statistically different between the 2 groups $(3.5 \pm 0.3$ and $3.2 \pm 0.3$ months, respectively). However, $11(92 \%)$ of 12 patients in the study group were detected at the time of the high-risk clinic visit or brought to our attention through HSM, which allowed for planned intervention. Only 1 patient came to our institution as a semiurgent transport from an outside hospital. Three of 5 patients who had demonstrated severely decreased right ventricular function were directly admitted from the high-risk clinic to our cardiac intensive care unit and underwent cardiac catheterization. One patient required angioplasty for then-identified recurrent arch obstruction, and the other 2 patients had no discernable anatomic lesion but had increased systemic vascular resistance. Both patients had completion of their S2P during that admission. In the control group 2 of 8 patients presented to an emergency department with clinical deterioration manifesting as poor feeding and perfusion, both of whom subsequently required arch angioplasty. One patient not suspected of any hemodynamic disturbances had detection of a significant arch obstruction at the time of pre-S2 catheterization and underwent angioplasty at that time.

Shunts. In the study group residual lesions identified after shunt placements included shunt and pulmonary artery stenosis $(n=1)$, arch obstruction $(n=1)$, failure to thrive $(\mathrm{n}=1)$, and severe ventricular dysfunction $(\mathrm{n}=1)$. In the control group lesions identified included shunt stenosis $(\mathrm{n}=5)$, discontinuous pulmonary arteries $(\mathrm{n}=1)$, arch obstruction $(\mathrm{n}=1)$, and right pulmonary artery stenosis $(\mathrm{n}=1)$. The mean age at detection, admission, and treatment of these lesions was younger in the study group (2.7 \pm 0.9 months) compared with that of control subjects $(6.6 \pm 0.6$ months, $P<.005)$. All residual lesions in the study patients were detected at the time of the high-risk clinic visit or brought to our attention through HSM. Two of 4 patients were direct admissions from the high-risk clinic to the cardiac intensive care unit. One patient with pulmonary atresia/intact ventricular septum had severe 
cardiac dysfunction on echocardiographic analysis and increased lactate levels on admission to the cardiac intensive care unit. It was decided to start this patient on cardiopulmonary support the same day. This patient underwent successful bidirectional cavopulmonary anastomosis (BDCPA) during that admission. The other patient, also with PAT/intact ventricular septum, had presented to our high-risk clinic with an oxygen saturation of $60 \%$ and was taken to the cardiac catheterization laboratory for stenting of both the shunt and left pulmonary artery.

In the control group the predominant lesion necessitating admission was shunt or pulmonary artery stenosis, occurring in 6 of 8 patients. The mean age at presentation was 6 months. All 6 patients presented with cyanosis, and 2 of 6 patients presented to outside emergency departments and required emergency transport to our hospital. Four of 6 patients were progressed semiurgently to BDCPA. One patient underwent stent implantation into the shunt, and 1 patient was electively scheduled for BDCPA. One of 8 patients had discontinuous pulmonary arteries detected at the time of pre-S2 catheterization, which was performed at 9 months of age. This patient had been previously lost to follow-up. He underwent successful BDCPA with reinstitution of pulmonary artery continuity.

\section{DISCUSSION}

The first quality improvement project undertaken by The Joint Council on Congenital Heart Disease Quality Improvement Task Force ${ }^{8}$ was directed toward improvement in the interstage quality of life for patients with HLHS between their S1P and S2P procedures. The concept of improvement in clinical outcomes through a reduction in institutional and individual cardiology practice variability is at the center of this project. Support for protocol-driven management associated with improved hospital survival and interstage survival is provided in many recent publications with regard to this fragile patient population. ${ }^{9}$ We know from other high-risk endeavors that it is exactly this checklist approach that is responsible for improvement in outcomes. In our experience a high-risk program leads to fewer interstage outpatient deaths and earlier detection of important recurrent/residual lesions.

A critical component to the success of any home intervention with high-risk patients is empowering the parent/ caregiver through education, repetition, and failsafe mechanisms. Parents must be enabled to contact their primary cardiologists, the tertiary center, or both at any time with a disturbance in the usual pattern of their infant. Hemodynamic compromise might manifest simply as feeding intolerance. A high-risk program offers a direct line of communication to a dedicated cardiac nurse practitioner who they know and with whom they have regular telephone contact. Any instruments we can provide to empower fam- ilies, whether the ability to call with a change in weight or a change in oxygen saturation, are key components in the success of HSM. Pulse oximetry and weight gain issues are familiar to parents after stays in the intensive care unit. Contact telephone numbers and printed materials that clearly delineate the patient's history and condition are also important. In particular, this material is important in the event of an emergency department visit or contact with medical personnel not familiar with single-ventricle physiology. The goal with this group of patients is to anticipate and detect problems early, thereby avoiding emergency department visits. The use of a database that is easily accessible and continually monitored helps ensure timely follow-up. On one occasion we used the Department of Social Services when we detected repeated missed appointments. All of the above mechanisms strive to minimize the stress imposed on families caring for infants with complex congenital heart disease.

In our experience minimizing the variation in care of these infants during the interstage period is of critical importance. This allows the team to provide anticipatory care. There were procedural changes in our congenital heart program that were a direct result of the high-risk program. We now have a scheduled time for pre-S2 catheterizations (3-4 months of age) for our patients with HLHS and a scheduled time for S2P (4 months of age). This was accomplished by scheduling the patient at the time of the high-risk visit for the catheterization and by the high-risk team facilitating the scheduling of subsequent operations. In the years before the high-risk program, palliated infants were catheterized anywhere from 4 to 8 months after their SIP, depending on individual practices. Not surprisingly, the older shunted infants manifested with problems of shunt stenosis and discontinuous pulmonary arteries. The timing of progression to $\mathrm{S} 2 \mathrm{P}$ should be institution dependent but should be consistent and provide a failsafe mechanism against loss of follow-up.

The ability to perform sedated echocardiography in a controlled and monitored environment with a dedicated sedation nurse and critical care physician gives the tertiary center an advantage over the private cardiology office. With few exceptions, sedated echocardiography provided the details necessary to identify many of the recurrent/residual lesions in these patients.

Recurrent arch obstruction was the most common reason for an interstage admission and catheter intervention in our patients with HLHS. What is not quantifiable is the detrimental effect of prolonged arch obstruction on cardiac function over the course of the patient's lifetime. In efforts to preserve long-term function, it is logical to assume that earlier detection and treatment is beneficial. For the palliated patients with shunts followed in our high-risk program, recurrent/residual lesions were detected at a significantly younger age. This suggests that a program such as this 
might be a more sensitive means for detecting residual lesions, leading to timely admission and treatment and possibly increased interstage survival. In the control group partial shunt occlusion was the most frequently identified lesion requiring intervention, yet only 1 patient presented with this problem in the study group. Perhaps with the earlier timing of our pre-S2 catheterization and progression to S2P there was less time for shunt occlusion to develop.

A high-risk program should be viewed as support for the private cardiologist during the most critical interstage period for these fragile patients. It should be designed to provide multidisciplinary care focused on medical, social, nutritional, and developmental needs. Frequent communication between parents and high-risk personnel helps identify potential problems early before the need for emergency care arises. Not uncommonly, our study patients had a lapse in health insurance coverage during this interstage period. This was relatively easy for our cardiac nurse practitioner to identify and our social worker to remediate. In the control group a lapse in insurance coverage might have contributed to loss or delays in follow-up care.

Our institution now offers hybrid palliation to a select group of patients with HLHS. Our first hybrid palliation was in 2007, 1 year after the inception of our high-risk program. All of our hybrid patients have been followed in our high-risk program. For the purposes of this article, these patients were not included because of the lack of a comparison group. Although beyond the scope of this discussion, it has been our experience that these patients might benefit the most from this type of intense interstage monitoring and support. These patients require more frequent high-risk outpatient evaluations. Although we have had no interstage deaths in this select population to date, all of our hybrid patients have had at least 1 and often multiple interstage admissions for catheter or surgical treatment of residual/ recurrent lesions.

\section{Limitations}

We were not able to follow all patients deemed eligible for our high-risk program because participation was at the discretion of the primary cardiologist. Our experience, in part, was a retrospective review, and our patient numbers are small. This supports the multicenter efforts to reduce the number of interstage mortalities in this fragile group of patients.

\section{CONCLUSIONS}

It is possible for a congenital heart program to attain nearly $0 \%$ interstage mortality in their most fragile infants after palliative operations. The mechanism for decreasing interstage attrition is multifactorial. We believe the key components revolve around empowering caregivers through education and failsafe mechanisms and enabling the cardiac team to provide as much anticipatory care as possible. The anticipatory care stems from minimizing variations in care. In our experience standardized interstage care for these infants allows for earlier detection and treatment of residual lesions, which might result in a significant reduction in morbidity and mortality.

\section{References}

1. Azakie T, Merklinger SL, McCrindle BW, Van Arsdell GS, Lee K, Benson LN, et al. Evolving strategies and improving outcomes of the modified Norwood procedure: a 10-year single-institution experience. Ann Thorac Surg. 2001;72: 1349-53.

2. Cua CL, Thiagarajan RR, Taeed R, Hoffman TM, Lai L, Hayes J, et al. Improved interstage mortality with the modified Norwood procedure: a meta-analysis. Ann Thorac Surg. 2005;80:44-9.

3. Simsic JM, Bradley SM, Stroud MR, Atz AM. Risk factors for interstage death after the Norwood procedure. Pediatr Cardiol. 2005;26:400-3.

4. Hehir DA, Dominguez TE, Ballweg JA, Ravishankar C, Marino BS, Bird GL, et al. Risk factors for interstage death after stage 1 reconstruction of hypoplastic left heart syndrome and variants. J Thorac Cardiovasc Surg. 2008;136:94-9, e1-3.

5. Ghanayem NS, Twedell JS, Hoffman GM, Mussatto K, Jaquiss RD. Optimal timing of the second stage of palliation for hypoplastic left heart syndrome facilitated through home monitoring, and the results of early cavopulmonary anastomosis. Cardiol Young. 2006;16(suppl 1):61-6.

6. Ghanayem NS, Hoffman GM, Mussato KA, Cava JR, Frommelt PC, Rudd NA, et al. Home surveillance program prevents interstage mortality after Norwood procedure. J Thorac Cardiovasc Surg. 2003;125:1367-77.

7. Dodds KM, Merle C. Discharging neonates with congenital heart disease after cardiac surgery: a practical approach. Clin Perinatol. 2005;32:1031-42, xi.

8. Kugler JD, Beekman RH, Rosenthal GL, Jenkins KJ, Klitzner TS, Martin GR, et al. Development of a pediatric cardiology quality improvement collaborative: from inception to implementation. From the joint council on congenital heart disease quality improvement task force. Congenit Heart Dis. 2009;4:318-28.

9. Srinivasan C, Sachdeva R, Morrow WR, Gossett J, Chipman CW, Imamura M, et al. Standardized management improves outcomes after the Norwood procedure. Congenit Heart Dis. 2009;4:329-37. 